CRÍTICA, Revista Hispanoamericana de Filosofia

Vol. XXII, No. 66 (diciembre 1990): 39-54

\title{
AZAR Y EXPLICACIÓN. ALGUNAS OBSERVACIONES *
}

\author{
ANA ROSA PÉREZ RANSANZ \\ Instituto de Investigaciones Filosóficas \\ Universidad Nacional Autónoma de México
}

El próposito de este trabajo es explorar algunas de las dificultades que enfrenta la explicación de los fenómenos azarosos. Con este fin, se discute primero el modelo clásico hempeliano para la explicación probabilística de sucesos singulares, destacando sus supuestos básicos y mostrando los problemasqque éstos generan. Posteriormente, se analizan algunas tesis de Wesley Salmon y se examina el modelo de Peter Railton - que constituyen propuestas alternativas frente a los problemas planteados por el modelo hempeliano-, para concluir con algunas observaciones acerca de los alcances y los límites de la explicación de los sucesos azarosos.

\section{I}

El modelo que Hempel propone para la explicación probabilística de sucesos particulares - llamado "estadístico-inductivo"- comparte con el modelo nomológico-deductivo los supuestos básicos de este autor acerca de la explicación científica. De acuerdo con la concepción hempeliana, la explicación consiste en subsumir, deductiva o inductivamente, aquello que

* Agradezco a Dorothy Edgington, Sergio Martínez, Raúl Orayen y Salma Saab sus valiosas críticas y observaciones a vensiones anteriones de este trabajo. 
se quiere explicar (el explanandum) bajo una o más leyes. De aquí la denominación de "modelo de cobertura legal [covering law model], o modelo de subsunción legal. De esta manera, se defiende una concepción inferencial de la explicación y se considera que toda explicación es un argumento.

Para Hempel, la tesis de que las explicaciones son argumentos lleva consigo la idea de que las explicaciones, sean deductivas o inductivas, nos muestran que el suceso por explicar es un suceso que era de esperarse [was to be expected] en virtud de ciertos hechos explicativos. Así, al referirse al modelo estadístico-inductivo, comparándolo con el nomológicodeductivo, Hempel afirma: “... aquí [en el esquema estadístico-inductivo] el explanans no implica lógicamente el explanandum; y el argumento no muestra que, asumiendo la verdad de los enunciados aducidos en el explanans, el fenómeno del explanandum era de esperarse 'con certeza'. Más bien, se puede decir que el argumento muestra que, con base en la información proporcionada por el explanans, el suceso del explanandum era de esperarse con certeza 'práctica', o con muy alta probabilidad" (Hempel 1965, p. 389). Como se puede ver, a pesar de sus diferencias, ambos modelos encierran la idea de esperabilidad [expectability]: el explanans muestra que el explanandum es algo esperable. Y en ambos casos se trata de una esperabilidad nómica: podemos esperar el explanandum en virtud de su inmersión bajo ciertas regularidades legales. Se podría decir entonces que en la concepción hempeliana las explicaciones explican justo al conferir esperabilidad nómica al explanandum.

Notemos que en las explicaciones probabilísticas, que Hempel intenta reconstruir con el modelo estadístico-inductivo, la esperabilidad tiene la connotación de alta probabilidad. Veamos por qué. En este tipo de explicaciones, cuyo explanans incluye de manera esencial al menos una ley estadística, resulta imposible contar con la certeza que ofrece la inferencia deductiva en relación con el suceso que se quiere explicar. Por tanto, 
para garantizar en estos casos que el explanandum siga siendo algo esperable en virtud del explanans, Hempel propone que las explicaciones probabilísticas se reconstruyan como argumentos inductivos con alta probabilidad. De esta manera, una explicación probabilística consta de leyes estadísticas y condiciones iniciales asociadas, las cuales conjuntamente confieren apoyo inductivo al suceso por explicar. (Cf. ibid. pp. 383-384). La fuerza o el grado en que se da este apoyo inductivo queda expresado por la probabilidad $r$ asociada a la explicación. Dice Hempel: "Por supuesto, un argumento de este tipo sólo será considerado explicativo si el número $r$ es bastante cercano a 1" (ibid., p. 390). Esto es, si se cumple el requisito de alta probabilidad.

La importancia que tiene la esperabilidad, en la teoría hempeliana, se pone claramente de manifiesto en la justificación que ofrece Hempel de la discutida tesis de que "toda explicación adecuada es potencialmente una predicción" (ibid., p. 367). Según Hempel, esta tesis encuentra su principal apoyo en un "principio general" que expresa una "condición de adecuación para toda explicación de sucesos particulares que sea racionalmente aceptable. Esa condición es la siguiente: cualquier respuesta racionalmente aceptable a la pregunta "¿por qué ocurrió el suceso $x$ ?' debe ofrecer una información que muestre que $x$ era de esperarse, si no de manera definitiva como en el caso de la explicación nomológico-deductiva, al menos con razonable probabilidad" (ibid., pp. 367-368). Como se puede ver, el principio general que expresa esta condición de adecuación no es otra cosa que la idea de esperabilidad.

Si bien lo anterior muestra el carácter fundamental que Hempel otorga a la esperabilidad, considero, sin embargo, que hay algo más detrás de esta idea. Veamos. Como dijimos, el requisito de alta probabilidad es exigido por Hempel para salvaguardar la esperabilidad en el campo de las explicaciones probabilísticas de sucesos particulares. La alta probabilidad garantiza que el suceso por explicar pueda ser esperado — inferido 
inductivamente- con "certeza práctica". Pero este requisito crea serias dificultades en la posición de Hempel: vuelve imposible la explicación de los sucesos poco probables, y esto genera a su vez una molesta asimetría. Supongamos que tenemos un proceso genuinamente estocástico —como el decaimiento alfa de un núcleo de uranio ${ }^{238}$ — donde hay dos posibles resultados, uno con muy baja probabilidad —que el núcleo emita una partícula alfa dentro de un cierto intervalo temporal-, y el otro con probabilidad muy alta —que no la emita. Resulta entonces que, de acuendo con el requisito de alta probabilidad, no podríamos explicar la ocurrencia de la emisión, pero sí podríamos explicar su no ocurrencia.

Frente a esta desconcertante situación, la pregunta que se plantea no es ya ¿̇or qué mantener el requisito de alta probabilidad?, sino, a un nivel más básico, ¿̇por qué tanto empeño en defender la esperabilidad? La respuesta, me parece, se encuentra en una intuición muy arraigada acerca de la explicación, intuición que está implícita en la concepción de Hempel, y que es la siguiente: aquello que explica la ocurrencia de un suceso $E$ no puede explicar también su no ocurrencia (o la ocurrencia de un suceso $E^{\prime}$ incompatible con $E$ ). Es decir, no puede ser que el mismo conjunto de circunstancias explique $E$ y $-E$. Otra manera de expresar esta intuición sería: cuando se explica un suceso particular dentro de un cierto marco teórico, se tienen razones en ese marco para descartar la ocurrencia de sus posibles alternativas; de lo contrario, no se estaría explicando. Llamaré a esta intuición principio básico.

Si se analiza con cuidado la condición hempeliana de adecuación arriba citada, se puede ver que la "respuesta racionalmente aceptable" a la pregunta ¿por qué ocurrió el suceso $x$ ?, satisface este principio básico al ofrecer información que muestra que $x$ era de esperarse (con certeza deductiva o con alta probabilidad). Cuando una explicación muestra la ocurrencia de un suceso como algo esperable, está descartando la ocu- 
rrencia de sus posibles alternativas, esto es, esta satistaciendo el principio básico.

Desde esta perspectiva se aprecia mejor la función que cumple el requisito de alta probabilidad. Si bien el principio básico se satisface plenamente en la explicación nomológico-deductiva (pues es imposible deducir dos enunciados incompatibles de un mismo conjunto consistente de premisas), sin embargo se ve seriamente amenazado en los argumentos de tipo estadístico (donde se infiere $E$ con probabilidad $r$ y se infiere $-E$ con probabilidad $1-r$, de un mismo conjunto de premisas). Frente a esta situación, la exigencia de alta probabilidad preserva el principio básico de la siguiente manera: dado que la suma de dos enunciados incompatibles no puede ser mayor que 1 , es imposible que ambos enunciados tengan alta probablidad; de este modo, si un argumento da cuenta de un enunciado con alta probabilidad, no puede al mismo tiempo dar cuenta de su negación.

Otro supuesto del modelo estadístico-inductivo, que también se desprende del principio básico, es el siguiente. Para Hempel, explicar es, en todos los casos, explicar por qué. Toda explicación científica es una respuesta a una pregunta por qué, sin importar cómo se formule de hecho la pregunta. Cuando Hempel analiza las preguntas sobre cómo es (o fue) posible la ocurrencia de un suceso, afirma que éstas simplemente revelan la perplejidad de quien hace la pregunta, es decir, revelan que las creencias previas de esa persona le hacen pensar que el suceso es imposible o altamente improbable. En este caso, la respuesta consistirá en hacerle ver lo equivocado de algunas de sus creencias, o bien en ampliar el campo de su evidencia de tal manera que el suceso en cuestión resulte altamente probable. (Cf. ibid., p. 429.) Una vez hecho esto, la pregunta original sobre cómo es posible se podrá reformluar como una pregunta por qué. Para Hempel, entonces, la diferencia entre preguntas por qué y preguntas cómo es posible se reduce a una diferencia de tipo pragmático. 
Sin embargo, como intentaré mostrar, existen situaciones, como las descritas en las teorías indeterministas, donde resulta totalmente inadecuado este análisis hempeliano de las preguntas sobre cómo es posible. En ese tipo de situaciones, preguntar por qué tienen lugar ciertos sucesos carecería de sentido; sólo son pertinentes preguntas sobre su posibilidad y éstas son irreformulables como preguntas por qué. De ser así, Hempel no estaría en lo cierto al afirmar que toda explicación científica responde a un por qué. Además, si Hempel hubiera reconocido la legitimidad de ciertas preguntas cómo - no reducibles a preguntas por qué-, hubiera podido salvar los escollos que le plantea la explicación de los sucesos poco probables. Me parece que el peso excesivo que Hempel otorga al principio básico le impidió contemplar esta vía.

Las explicaciones de por qué y las explicaciones sobre cómo es posible, responden a requerimientos epistémicos distintos, independientes e igualmente legítimos. Intentando una caracterización mínima, podríamos decir que las explicaciones por qué deben dar cuenta de la ocurrencia de un suceso eliminando sus posibles alternativas (deben cumplir el principio básico), mientras que las explicaciones sobre cómo es posible deben dar cuenta de la ocurrencia de un suceso mostrando que su probabilidad no es nula. Esta distinción, como se puede ver, restringe el campo de aplicación, y por tanto la validez, del principio básico. Consideraciones como el marco teórico del cual se parta y los objetivos de cada investigación, indican qué tipo de explicación es el pertinente en cada caso.

Lo anterior parece indicar que la pretensión de Hempel de explicar probabilísticamente sucesos específicos, es una pretensión demasiado fuerte. El principio básico —con su consecuente exigencia de esperabilidad y alta probabilidad_-, más el supuesto de que toda explicación es una explicación de por $q u e ́$, hacen que el modelo estadístico-inductivo enfrente, de manera insuperable, dificultades como las señaladas: la imposibilidad de explicar la ocurrencia de los sucesos poco pro- 
bables y la inaceptable asimetría que esto acarrea. Estas dificultades se podrían evitar rechazando en general el principio básico - lo cual volvería innecesario el requisito de alta probabilidad-, o negando que las explicaciones probabilísticas de sucesos particulares puedan ser explicaciones de por $q u e ́$. Veremos esto con más detalle.

\section{II}

Wesley Salmon adopta la primera de las vías recién señaladas. Siguiendo a Richard Jeffrey, este autor postula un principio de simetría: "Cuando un proceso estocástico da lugar a ciertos resultados que son altamente probables y a otros que son improbables, comprendemos los improbables tan bien (o tan pobremente) como los probables" (Salmon 1989, pp. 178-179). Claramente este principio de simetría implica el rechazo del principio básico pues permite que el mismo conjunto de circunstancias explique la ocurrencia de sucesos incompatibles. Sin embargo, Salmon considera que: "El mostrar que un resultado es altamente probable, y que era de esperarse, no tiene nada que ver con la explicación" (ibid., p. 62). Para él, la clave de toda explicación científica, y en particular de las explicaciones probabilísticas de sucesos singulares, está en comprender los mecanismos responsables: "El objetivo [de la explicación] es mostrar las formas en que opera la naturaleza; es un esfuerzo por descubrir los mecanismos que subyacen a los fenómenos que observamos y deseamos explicar" (Salmon 1990).

La posición de Salmon refleja una manera básicamente distinta de la hempeliana de entender la explicación. La obligada elección entre el principio de simetría y el principio básico, dada su incompatibilidad, determina perspectivas muy ajenas entre sí. Veamos con más cuidado las implicaciones de optar por el principio de simetría.

Como bien dice Salmon: "En este momento de la historia de la física, nuestras teorías filosóficas sobre la explicación 
deben dejar abierta la posibilidad de que el mundo sea indeterminista" (Salmon 1989, p. 173). La razón principal es que la mecánica cuántica, que es una teoría indeterminista, "ha tenido más éxitos explicativos que cualquier otra teoría en la historia de la ciencia" (ibid.). Pero entonces, al asumir que el mundo es tal como lo describen nuestras mejores teorías físicas, tenemos que admitir sucesos cuya ocurrencia es genuinamente azarosa. Esto hace surgir la siguiente duda: ¿el que la ocurrencia específica de un suceso sea realmente una cuestión de azar, acaso no señala un cierto límite en nuestra capacidad explicativa?

Cuando Salmon afirma que, dado un proceso estocástico, podemos comprender igualmente bien la ocurrencia y la no ocurrencia de un mismo suceso - basándose en el principio de simetría-, está suponiendo que podemos explicar las ocurrencias azarosas particulares. Este 'explicar' requiere aclaración. Me parece que dada una teoría indeterminista podemos entender - con mucha dificultad, despúes de siglos de una visión laplaciana determinista- que existan mecanismos y procesos estocásticos, gobernados por leyes irreductiblemente probabilísticas, los cuales dan lugar indistintamente a sucesos incompatibles, es decir, sin que medie ningún cambio en las circunstancias dadas. Esto quiere decir que aceptamos que las probabilidades en estos casos no son el resultado de nuestra ignorancia, pues no falta ningún componente explicativo ni en el nivel de las leyes ni en el nivel de los hechos. Suponemos, por tanto, que la mecánica cuántica es una teoría completa con respecto a su campo de estudio. Sin embargo, esto no nos compromete con la tesis de que podemos explicar, en el sentido de explicar por qué, cada una de las ocurrencias de los sucesos del nivel cuántico.

Al discutir la explicación de las ocurrencias azarosas, y postular el principio de simetría, Salmon no distingue entre explicar por qué y explicar cómo es posible. Me parece que esta distinción cobra especial importancia en el contexto de las teorías 
indeterministas, pues permitiría precisar el principio de simetría de la siguiente manera: dado un mecanismo estocástico, podemos comprender igualmente bien los resultados altamente probables y los poco probables en el sentido de que podemos explicar cómo son posibles. El principio de simetría sólo tiene validez en las explicaciones que aducen condiciones de posibilidad —como serían los mecanismos estocásticos-, pues es sólo en este caso que se puede ofrecer la misma explicación para ocurrencias incompatibles.

La explicación de los fenómenos azarosos recibe un tratamiento muy iluminador en los trabajos de Peter Railton. Para este autor "la explicación propiamente llamada probabilística es la explicación de las cosas que suceden por azar: los resultados de los procesos irreductiblemente probabilísticos" (Railton 1981, p. 233). Por tanto, los fenómenos que comúnmente se han considerado objeto de explicación probabilística —los resultados de juegos de azar, los fenómenos de la mecánica estadística y la termodinámica clásicas, los pronósticos del tiempo, etc.- , pero que no son fenómenos genuinamente indeterministas, no caen bajo el modelo probabilístico que Railton propone. Sin embargo, este autor reconoce que: "El uso de probabilidades epistémicas o estadísticas en conexión con tales fenómenos tiene indudablemente un valor instrumental, y no deberían abandonarse" (Railton 1978, p. 223).

Railton considera que los problemas que enfrenta el modelo hempeliano se derivan de su carácter inductivo: "Si se ofrece un modelo no inductivo para la explicación estadística de hechos particulares, no hay necesidad de caer en la tentación de pedir alta probabilidad o de excluir la verdad" (ibid., p. 212). El modelo deductivo que propone Railton - llamado "nomológico- deductivo-probabilístico" (DNP) — es elaborado a patir del análisis de un ejemplo, el ejemplo de un suceso que tiene una probabilidad extremadamente pequeña: el decaimiento alfa de un núcleo de uranio ${ }^{238}$. Consideremos el siguiente argumento deductivo: 
I (a) Todos los núcleos de $\mathrm{U}^{238}$ tienen la probabilidad $p$ de emitir una partícula alfa durante cualquier intervalo de longitud $\tau$, siempre y cuando no estén sujetos a radiación ambiental.

(b) $u$ era un núcleo de $\mathrm{U}^{238}$ en el tiempo $t_{0}$ y no estuvo sujeto a radiación ambiental durante el intervalo $t_{0}-\left(t_{0}+\tau\right)$

(c) $u$ tenía una probabilidad $p$ de decaimiento alfa durante el intervalo $t_{0}-\left(t_{0}+\tau\right)(C f$. Railton 1981, p. 235).

Este argumento constituye una explicación nomológico-deductiva (en el sentido hempeliano clásico) del hecho de que u tuviera una determinada probabilidad de decaimiento alfa en el intervalo en cuestión. Para llegar a tener una explicación del decaimiento ocurrido - de que $u$ haya emitido de hecho la partícula alfa_ es necesario, según Railton, complementar el anterior argumento con los siguientes elementos: $(l)$ una derivación de $I(a)$ "a partir de nuestra explicación [account] teórica del mecanismo que opera en el decaimiento alfa" (Railton 1978, p. 214); y (2) un "añadido entre paréntesis" [parenthetic addendum] que diga que, en efecto, el decaimiento tuvo lugar.

Como se puede ver, una explicación DNP de la ocurrencia específica de un suceso azaroso, debe cumplir tres requisitos:

- dar cuenta del mecanismo indeterminista en juego (de lo cual se deriva la ley probabilística en cuestión),

- atribuir una probabilidad determinada al suceso (derivándola de la ley probabilística), y

- afirmar la ocurrencia efectiva del suceso ("añadido entre paréntesis").

En el caso analizado, Railton afirma que la derivación de 
$I(a)$ es posible porque el decaimiento alfa está gobernado por leyes físicas, tanto pmóabilísticas (como las leyes que se refieren a la penetración de barreras [barrier penetration] y algunos otros fenómenos cuánticos), como deterministas (las leyes de conservación de la masa-energía, de la carga, del momento, etc.). Esto significa que el azar postulado por las teorías físicas es un azar legal.

Ahora bien, en relación con el argumento $I$ me surge una duda con respecto a la formulación de la ley $I(a)$. Aun cuando en este caso fuera correcto reconstruir el enunciado como una generalización universal: todos los $A$ tienen la probabilidad $p$ de $\operatorname{ser} B$, no es evidente que toda ley estadística pueda o deba formularse de esa manera. Cuando una ley estadística se formula como: la probabilidad de que los $A$ sean $B$ es $p$, no es susceptible de instanciación universal y, por tanto, no podría (por modus ponens) dar lugar a una conclusión acerca de la probabilidad de un suceso o caso singular. Para este tipo de leyes estadísticas, entonces, el modelo de Railton resultaría inaplicable.

Pasemos ahora al problemático requisito del "añadido entre paréntesis", es decir, de la información de que el suceso por explicar efectivamente ocurrí. En su trabajo de 1978, Railton considera el añadido como una "parte crucial de la historia" ya que: "El abandonar el añadido deja una explicación, pero una explicación nomológico-deductiva de la ocurrencia de una probabilidad particular, no una explicación probabilística de la ocurrencia de un decaimiento particular" (ibid., p. 217). El añadido, entonces, parece ofrecer una información indispensable para la explicación de ocurrencias azarosas.

Notemos que este añadido no se puede agregar al argumento I como una premisa más, pues estaríamos agregando justo lo que queremos explicar y el argumento se volvería trivialmente circular. Esta es la principal razón del rechazo de Railton a la tesis hempeliana de que toda explicación es un argumento -tesis que Salmon denomina "el tercer dogma del empiris- 
mo"- - ya que el añadido es necesario para la explicación. Notemos también que el añadido, en tanto requisito, indica que la explicación DNP sólo se puede dar ex post facto. Las cuestiones de azar, dice Railton, "se resisten a todo menos a la demostración ex post facto" (ibid. p. 209). Esto implica claramente el rechazo de otra tesis hempeliana, la idea de esperabilidad.

Ahora bien, frente a esta propuesta uno se pregunta: qué información relevante puede aportar el añadido, o qué fuerza explicativa se gana con saber que el suceso por explicar en efecto ocurrib. El contraste con lo que sucede en el campo de las teorías deterministas puede ser iluminador. La utilización de leyes estadísticas en la explicación de fenómenos que se consideran deterministas -explicaciones que, como vimos, Railton no considera genuinamente probabilísticas- se debe a una falta de conocimiento o de control sobre todas las condiciones iniciales. En esta situación, puede suceder que la información sobre cuál de los posibles resultados es el que en efecto se dio, sirva para construir una explicación más completa de ese mismo resultado; incluso podría permitir dar una explicación de por qué se dio ese resultado, aunque sólo de manera ex post facto. Este podría ser el caso de la determinación genética de los caracteres heredados. Por tanto, en el campo determinista el conocimiento de la ocurrencia de un suceso puede ser útil o relevante cuando entran en juego leyes estadísticas.

En cambio, cuando se parte de leyes irreductiblemente probabilísticas y se trata con sucesos genuinamente azarosos, la información sobre las ocurrencias efectivas no permite agregar ningún factor explicativo más. Según Railton, la información que aporta el añadido "es relevante para el origen causal del explanandum, al decimos que llego a darse como la realización de una posibilidad física particular" (ibid., p. 217). Sin embargo, me parece claro que esto no nos lo dice el añadido sino que lo sabemos, precisamente, (1) al dar cuenta del mecanismo estocástico en juego, y (2) al saber que el explanandum tiene 
cierta probabilidad, no nula, de ocurrir. Es decir, lo sabemos por los componentes (1) y (2) del modelo DNP sin el añadido.

Notemos, entonces, que al eliminar el añadido del modelo DNP nos queda: (a) una explicación de cómo es posible la ocurrencia de un cierto suceso azaroso - apelando al mecanismo que lo genera con una probabilidad no nula-, y al mismo tiempo (b) una explicación de por qué ese suceso tiene la probabilidad que tiene de ocurrir y no otra - ya que se deducen valores de probabilidad bien definidos. En el campo de los fenómenos azarosos, estos dos aspectos conformarían el objetivo de las explicaciones probabilísticas. No hay nada más que se pudiera explicar con respecto a la ocurrencia de este tipo de sucesos. Entendida así la explicación probabilística, es claro que no tiene por qué ser ex post facto.

Si bien es cierto que en su artículo de 1981, Railton admite que el añadido podría ser dispensable -a pesar de lo cual decide seguir manteniéndolo (Cf. Railton 1981, p. 236)—, el interés en discutir el modelo DNP en su versión original ha sido destacar algunos rasgos distintivos de la explicación en las teorías indeterministas. Por otra parte, el modelo DNP — sin el carácter ex post facto- nos permite aplicar de una manera muy natural la distinción entre explicar cómo y explicar por qué (aunque Railton tampoco hace explícita esta distinción).

La utilidad de tomar este modelo haciendo explícita la distinción es la siguiente. Por un lado, nos permite ver que no es necesario rechazar totalmente el principio básico, como propone Salmon, pues este principio sigue teniendo validez en la explicación de las probabilidades: según este modelo, podemos explicar por qué un suceso tiene la probabilidad que tiene de ocurrir y no otra. (Como señalamos antes, esta asignación de probabilidades a sucesos singulares es posible siempre y cuando las leyes estadísticas se puedan formular como generalizaciones universales.) Por tanto, con respecto a la explicación de las probabilidades no sería aplicable el principio de simetría defendido por Salmon. 
Por otro lado, podemos ver que el principio básico tampoco es universalmente aplicable, como pretende Hempel. Tratándose de procesos genuinamente estocásticos, no hay razón alguna que se pueda aducir en favor de la ocurrencia de uno de los resultados, descartando el (los) otro(s). Se trata de una imposibilidad de principio. Por tanto, carecería de sentido preguntar por qué ocurrió tal cosa y no otra; esto equivaldría a pedir que el azar no fuera azaroso. Si tomamos es serio el azar del que nos hablan nuestras mejores teorías físicas, debemos aceptar que hay preguntas por qué que simplemente no tienen respuesta, o cuya única respuesta posible es: por azar. Esto significa que tenemos que reconocer un cierto límite en nuestras capacidades explicativas.

Sin embargo, no deja de resultar asombroso que haya respuesta a las preguntas sobre cómo es posible el azar, es decir, que se conozcan los mecanismos que lo generan y las leyes que lo rigen. La precisión con la que se puede calcular la probabilidad de que ocurran los sucesos azarosos, no tiene precedentes en la historia de la ciencia. Curiosamente, las teorías indeterministas han resultado hasta la fecha nuestras teorías más exitosas.

En conclusión, el modelo de Railton — sin el carácter ex post facto- nos permite reconocer con bastante claridad los alcances y los límites de la explicación probabilística de los sucesos azarosos. Al hacer explícita en este modelo la distinción entre explicar por qué y explicar cómo, podemos ubicar el principio básico y el principio de simetría en el lugar que les corresponde, recuperando de esta manera el núcleo de verdad que encierran las concepciones de Hempel y de Salmon sobre la explicación científica.

\section{BIBLIOGRAFIA}

Hempel, Carl G. [1965]: “Aspects of Scientific Explanation”, Aspects 
of Scientific Explanation and Other Essays in the Philosophy of Science, Nueva York: The Free Press, pp. 331-496.

Railton, Peter [1978]: “A Deductive-Nomological Model of Probabilistic Explanation", Philosophy of Science 45, pp. 206-226.

Railton, Peter [1981]: “Probability, Explanation, and Information", Synthese 48, pp. 233-256.

Salmon, Wesley [1984]: Scientific Explanation and the Causal Structure of the World, Princeton: Princeton University Press.

Salmon, Wesley [1989]: "Four Decades of Scientific Explanation" en W. Salmon y P. Kitcher (eds.), Scientific Explanation, Minnesota Studies in the Philosophy of Science, Vol. XIII, Minneapolis: University of Minnesota Press, pp. 3-219.

Salmon, Wesley [1990]: "Scientific Explanation: Causation and Unification”, publicado en este número de Crítica, pp. 3-21.

Recibido: 2 actubre 1990. 


\section{SUMMARY}

In the first part of this paper I examine the Hempelian model for the probabilistic explanation of particular events: the inductivestatistical model. Here I focus on an examination of the notion of expectability and the implied requirement of high probability. I intend to show that expectability and high probability, in turn, answer to a deep rooted intuition concerning explanation: given an event $E$, the same sort of circumstances cannot explain $E$ and $-E$. This intuition is called here "the basic principle".

This basic principle.is also the ground for the Hempelian thesis that to explain is always to explain why. I think this thesis is wrong and I propose to distinguish between explaining why and explaining how possibly. I discuss the difficulties confronting the statisticalinductive model in the light of the assumptions here examined and suggest possible solutions.

In the second part, I explore those possible solutions, in particular I examine the symmetry principle proposed by Salmon: given an stochastic process, the highly probable results are as well understood as the improbable results. This principle implies the rejection of the basic principle. I claim that the symmetry principle must be restricted to explanations how. Later I discuss the DNP model proposed by Railton and I make some criticisms to it. I try to show that if we make explicit the distinction between explaining why and explaining how in this model, we can restrict clearly the range of applicability of the symmetry principle and the basic principle. I conclude that Railton's model constitutes an adequate basis to establish the scope and limits of probabilistic explanations of genuinely random events. 